\title{
Early Outcome of Discectomy with Interspinous Process Distraction Device a Retrospective Cross- Sectional Study
}

\author{
Gunaseelan Ponnusamy* \\ Nasional Universty of Malaysia, Malaysia \\ *Corresponding author: Gunaseelan Ponnusamy, 1938 Jalan G-1, Taman Melawati 53100 Kuala Lumpur, Malaysia \\ Submission: 酒 February 25, 2018; Published: 海 March 21, 2018
}

\begin{abstract}
Objectives: The main aim of this retrospective study was to evaluate the usefulness and early outcome and radiological changes after discectomy with DIAM implant insertion for patients with herniation of nucleus pulposus.

Methods: Thirty-three patients underwent discectomy with DIAM implant insertion for a herniation of nucleus pulposus between June 2009 and April 2014 in Hospital Kuala Lumpur, Malaysia were considered for this study. All datas were collected by reviewing the patient's medical record. All patients had back pain and leg or buttock pain associated with radiological evidence of herniation of nucleus pulpous with failure to 3 months of conservative management. All patients had post-operative follow-up at 2 weeks, 3 months and 6 months after the surgery with documented VAS scores (back and leg pain) and Oswestry Disability Index preoperative and postoperatively. Disc height was measured on the preoperative and postoperative plain radiograph.
\end{abstract}

Results: 23 male and 10 female were treated. The mean age was 39.82 years old. The most common level was L5/S1 (58\%). Preoperative VAS score for leg pain and low back pain improved from $4.3 \pm 1.7$ and $4.5 \pm 1.4$ to $1.1 \pm 0.8$ and $2.2 \pm 1.2$ respectively at last clinic visit $(\mathrm{p}<0.001)$. The average preoperative ODI was $24.6 \pm 18.1$ and postoperative at 6 -month was $12.5 \pm 10.2$ giving a statistically significant difference ( $<<0.001$ ) in functional disability outcome. There was no significant in preoperative and postoperative disc height and intervertebral angle at index level.

Conclusion: The early outcome of treatment of low back pain with radiculopathy treated with discectomy and placement of non-fusion DIAM interspinous process spacer showed significant ODI and VAS outcome scores at baseline, 2 weeks, 3 months and at 6 months follow up. There were no significant changes in preoperative and postoperative disc height and intervertebral angle at index level. No adverse local or systemic reaction to the DIAM was noted.

Keywords: Interspinous spacer; Herniation nucleus pulposus; Clinical outcome

\section{Introduction}

Posterior spinal diseases resulting from lumbar disc herniation or lumbar spinal stenosis is associated with significant structural failure of intervertebral disc, of the ligaments, and/or of the bone structures [1]. The typical failures includes disc prolapses, endplate damage, internal disc disruption disc space narrowing, hypertrophic facet joints, hypertrophic ligaments and osteophytes. These degenerative changes may cause instability in advanced stages of the disease. The clinical endpoint of these degenerations is the compression of neural structures at the level of neural foramina or of the spinal canal.

Patients will typically complain of low back pain with or without radicular pain or dysesthesia. The operative "gold standard" of treatment of degenerative lumbar spinal disease is decompression with or without fusion of the affected segment. Improvements in surgical techniques and implants have improved the successful fusion rate but not have significantly improved the clinical outcome. This observation has led to increasing interest in motion preservation techniques in the treatment of low back pain.

The decision to perform discectomy with insertion of interspinous process distraction (IPD) device in patients with posterior spinal diseases is controversial [2,3]. Although the insertion of of an IPD device has become a relatively common procedure for the treatment of lumbar disc herniation or lumbar spinal stenosis, its concomitant use with discectomy is not clear given the lack of comparative studies [4,5]. IPD devices represents a relatively new option of treatment as an alternative to conventional 
decompressive surgery in managing symptomatic lumbar spinal pathology [6]. These devices has mechanical goal of distracting the interspinous space thus increasing intervertebral space height and are designed for placement between adjacent spinous processes. They act mainly on the posterior part of functional spinal unit by distracting the spinous processes and avoiding extension of the treated segment. It also indirectly increases the cross-sectional area of spinal canal as well as the neuroforamina. The reported treatment indications are variable, ranging from treatment of degenerative spinal stenosis, discogenic low back pain, facet syndrome, disc herniations, and instability [3].

In Malaysia, there is lack of any outcome study of patients with lumbar posterior spinal diseases treated with IPD device. We selectively look into patients with low back pain with radiculopathy from prolapsed intervertebral disc treated with discectomy and IPD device. The IPD device involved in this study is DIAM (Device of Intervertebral Assisted Motion) by Medtronic Safamor Danek. We therefore performed the current study to determine the early outcome of patients treated with discectomy and DIAM implant insertion.

\section{Methodology}

This retrospective study was performed in Hospital Kuala Lumpur from June 2009 to April 2014. This study was approved by Ministry of Health Research and Ethics Committee Malaysia.

\section{Patient selection}

Inclusion criteria were as follows. Patients with age greater than 18 years old. Patients must have attempted and failed at least 3 months of conservative therapy, such as physical therapy and analgesics (oral steroids, non steroidal anti-inflammatory drugs NSAIDS). All patients must have had lumbosacral plain radiographs done at initial presentation. All patients carried a diagnosis of prolapsed intervertebral disc that were confirmed by MRI. Patients were all considered surgical candidates with a disease severity justifying a discectomy and DIAM implant insertion. All patients must have had VAS for leg and back pain at preoperative and postoperatively at 2 weeks, 3 months and/or 6 months. and Oswestry Disability questionnaire filled preoperative and postoperatively at 6 months.

Exclusion were as follows. Patients with any previous history of any spine surgery, with significant instability (e.g. spondylolisthesis greater than Grade 1), significant scoliosis (Cobb angle is greater than 250), degenerative neurologic disease, an ankylosed segment at the affected level, a history of previous spinous process fracture or pars interarticularis fracture, known peripheral neuropathy, cauda equina syndrome, on-going spinal or systemic infection were excluded from this study.

\section{Surgical procedure}

The device were implanted after discectomy under general anaesthesia and with the patient lying in the prone position. Patients are required to maintain a flexed position to aid in distraction of the spinous process. Surgical levels are first correctly identified using fluroscopy. The soft tissue is dissected to the fascia and the fascia is incised longitudinally to the right and left of the midline. The appropriate surgical levels is then reverified via fluroscopy. Discectomy was performed on all patients. The interspinous space were prepared by squaring it off with a ronguer. The space is then distracted and measuring trials were used to determine the size of the DIAM. The implant is inserted and seated with a tamp and mallet. The attached cords are wrapped around the spinous processes above and below the DIAM implant, tightened and then crimped. Proper positioning of the implant is verified using fluoroscopy prior to closure. Wound was irrigated and fascial closures were used to approximate the facial incisions prior to standard closure of subcutaneous and skin layers. Patients are typically discharged from the hospital 2 days after the surgery.

\section{Assessment of clinical outcome}

The main outcome measurements used in this study are Visual Analogue Score (VAS) for pain and Oswestry Disability Index (ODI) for functional outcome. VAS refers to a unidimensional measure of pain intensity [7]. Typically patients are asked to report "current" pain intensity or pain intensity "in the last 24 hours" of the lower back and leg pain. The VAS score is self-completed by the patient. Higher score indicated higher pain intensity. ODI is a selfadministered questionnaire divided into ten sections designed to assess limitations of various activities of daily living. The test has been around for more than 25 years and is considered the "gold standard" of low back functional outcome tool. It simply answers some questions by choosing the "best answer" that describes the "typical" pain and/or limitations within the last week or two. ODI scoring $0 \%$ to $20 \%$ means minimal disability (patient can cope with most living activities, no treatment indicated apart form exercise), 21\%-40\% moderate disability (patient experiences more pain and difficulty with sitting, lifting and standing, patient managed by conservative means), 41\%-60\% severe disability (pain remains the main problem, activities of daily living are affected, require a detailed investigation), 61\%-80\% crippled (back pain impinges on all aspects of patients life, intervention is required), and 81\%$100 \%$ are either bed-bound or exaggerating their symptoms [8].

\section{Radiological measurements}

For radiological measurements, we calculated the mean disc height and intervertebral disc angle at index level, and adjacent disc height and disc angle. Preoperative and postoperative radiographs of lumbosacral are done. Disc height at index level and adjacent level are measured based on the modified Farfan method (Figure 1) [9]. The measurement is done by first drawing a line from the anteroinferior corner of the superior vertebrae (A) to posteroinferior corner of the superior vertebra (B). A second line are drawn from the anterosuperior corner of the inferior vertebrae (C) to posterosuperior corner of the inferior vertebrae (D). Four perpendicular lines are drawn from each edges of the superior and inferior vertebras (H1,H2,h1,h2). Disc height are then measurement by the sum of $\mathrm{H} 1+\mathrm{H} 2+\mathrm{h} 1+\mathrm{h} 2$ and dividing it by 
4. Measurement of intervertebral disc angle are done by drawing a line along the inferior end plate of the superior vertebra and another line drawn along the superior end plate of the inferior vertebra.10 The angle between this two lines are taken as the disc angle. All the measurement are done by one researcher to reduce inter-observer error. A total of 33 patients presented with leg pain, with 8 patients also describing low back pain. All patients had no history of previous spine surgery prior to DIAM implant insertion. All patients had previously received conservative treatment mainly physical therapy and most patients had taken analgesia like non- steroidal anti-inflammatory drugs for back pain and leg pain. The mean age in this study was 40 years old, with the youngest at 25 years old and oldest at 70 years old. The majority of patients were male (69.7\%), and based on ethnicity, Malays contributed $72.7 \%$ of cases followed by Chinese (15.2\%), Indians (6.1\%) and others (6.1\%) (Table 1). A total of 33 DIAMs were inserted in 33 patients. The underlying disorders were due to prolapsed intervertebral disc. The most common level of insertion was L5/S1 at 59\%, followed by L4/L5 level at 41\% (Figure 2).

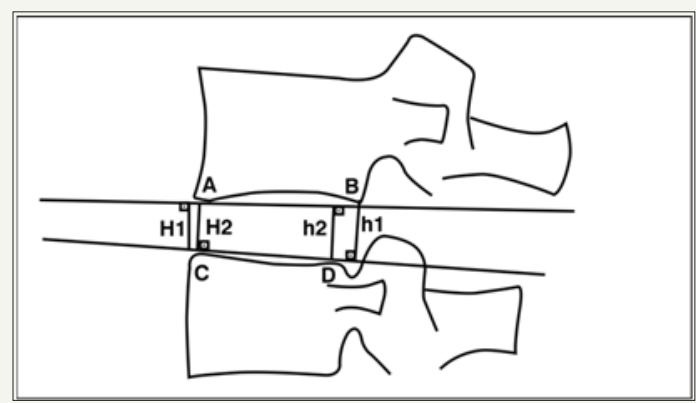

Figure 1: Measurement of intervertebral disc height based on modified Farfan method9.

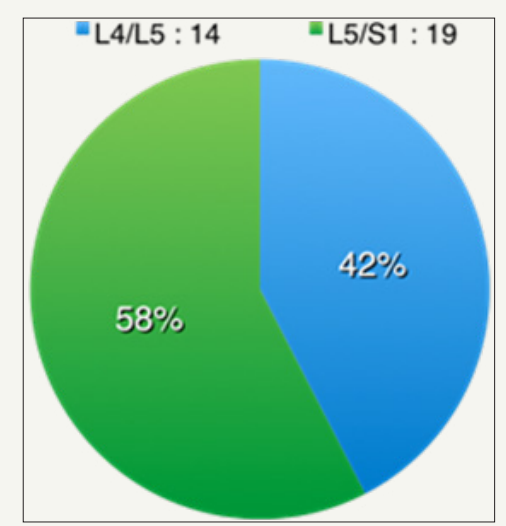

Figure 2: Measurement of intervertebral disc height based on modified Farfan method9.

Table 1: Demographic distribution of subjects.

\begin{tabular}{|c|c|}
\hline Characteristics & Number (Percentage) n (\%) \\
\hline Number of cases & 33 \\
\hline Age (Years) & 40 \\
\hline Median (IQR) & $(25.00,70.00)$ \\
\hline Gender: & $24(69.7)$ \\
\hline Male & $10(30.3)$ \\
\hline Female & \\
\hline Ethinicity: & $23(72.7)$ \\
\hline Malay & $5(15.2)$ \\
\hline Chinese & $2(6.1)$ \\
\hline Indian & $2(6.1)$ \\
\hline Others & \\
\hline
\end{tabular}

\section{Leg pain}

The mean VAS preoperative leg pain was $4.3( \pm 1.74)$ in 33 patients (Table 2). The mean pain values at 2 weeks, 3 months and 6 months significantly dropped to $3.04,1.65$ and 1.09 respectively.
Using Wilcoxon Signed Rank test, there is a highly significant difference between each time frame of VAS score (Figure $3 \& 4$ ).

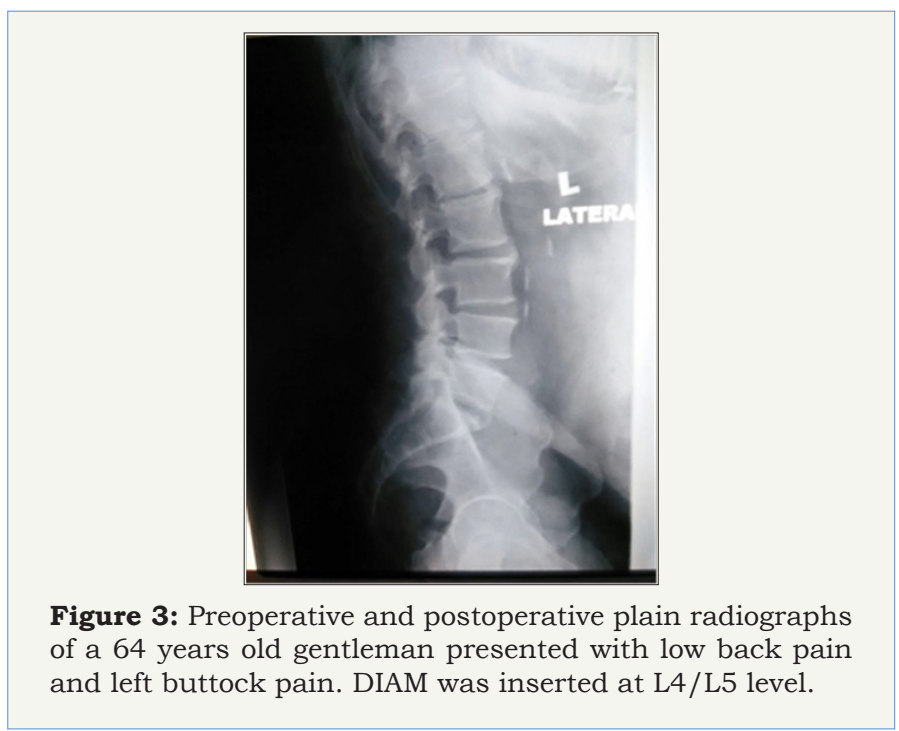


Table 2: Mean and descriptive results of VAS leg score at pre-op, 2 weeks, 3 months and 6 months.

\begin{tabular}{|c|c|c|c|c|}
\hline LEG (n=33) & Mean & Std. Deviation & Min & Max \\
\hline VAS pre-op & 4.3 & 1.74 & 1 & 5 \\
\hline VAS at $2 / 52$ & 3.04 & 1.08 & 1 & 3 \\
\hline VAS at 3/12 & 1.65 & 0.7 & 0 & 2 \\
\hline VAS at 6/12 & 1.09 & 0.77 & 0 \\
\hline
\end{tabular}

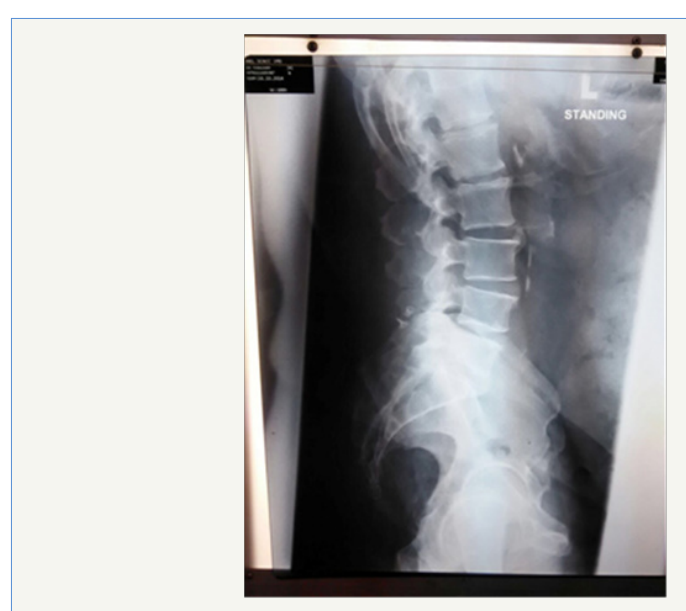

Figure 4: Preoperative and postoperative plain radiographs of a 64 years old gentleman presented with low back pain and left buttock pain. DIAM was inserted at L4/L5 level.

\section{Back pain}

The mean VAS preoperative back pain score was 4.50 in 8 patients (Table 3). The mean pain values at 2-weeks, 3 months and 6 months dropped to 3.86, 2.20 and 2.17. Using Wilcoxon Signed Rank test, there is a highly significant difference between prep

Table 3: Mean and descriptive results of VAS back score at pre-op, 2 weeks, 3 months and 6 months.

\begin{tabular}{|c|c|c|c|c|}
\hline BACK $(\mathbf{n = 8})$ & Mean & Std. Deviation & Min & Max \\
\hline VAS pre-op & 4.5 & 1.41 & 2 & 6 \\
\hline VAS at $2 / 52$ & 3.86 & 1.07 & 1 & 4 \\
\hline VAS at $3 / 12$ & 2.2 & 1.3 & 1 & 4 \\
\hline VAS at $6 / 12$ & 2.17 & 1.17 & \\
\hline
\end{tabular}

Table 4: Mean and descriptive results of Pre-op and Post-op ODI score.

\begin{tabular}{|c|c|c|c|c|}
\hline ODI (n=33) & Mean & Std Deviation & Minimum & Maximum \\
\hline Pre-op & 24.59 & 18.07 & 4 & 72 \\
\hline Post-op & 12.46 & 10.16 & 0 & 36.6 \\
\hline
\end{tabular}

Table 5: Mean and Standard Deviation of Pre-op and Post-op Disc Height.

\begin{tabular}{|c|c|c|c|c|}
\hline Disc Height IL & N & Mean & Std. Deviation & Std. Error Mean \\
\hline Pre-op & 17 & 11.02 & 1.75 & 0.42 \\
\hline Post-op & 17 & 10.87 & 1.69 & 0.41 \\
\hline
\end{tabular}

Table 6: Mean and Standard Deviation of Pre-op and Post-op Intervertebral (IV) Disc Angle.

\begin{tabular}{|c|c|c|c|c|}
\hline IV Disc Angle & N & Mean (0) & Std. Deviation & Std. Error Mean \\
\hline Pre-op & 17 & 9.24 & 2.91 & 0.71 \\
\hline Post-op & 17 & 9.65 & 2.15 & 0.52 \\
\hline
\end{tabular}




\section{Adjacent level disc Hheight}

Out of the 33 patients, only 17 patients data were able to be collected for analysis. Based on Kolmogorov test for normality, it is noted that the preoperative and postoperative disc height is

Table 7: Mean and Standard Deviation of Pre-op and Post-op Adjacent Level Disc Height.

\begin{tabular}{|c|c|c|c|c|}
\hline Adjacent Disc Ht & N & Mean & Std. Deviation & Std. Error Mean \\
\hline Pre-op & 17 & 11.33 & 2.57 & 0.62 \\
\hline Post-op & 17 & 11.04 & 1.77 & 0.43 \\
\hline
\end{tabular}

Adjacent Level Disc Angle: Out of the 33 patients, only 17 patients data were able to be collected for analysis. Based on Kolmogorov test for normality, it is noted that the preoperative and postoperative disc height is normally distributed. Thus a parametric test is used for analysis. Using t-test, $p=0.02$. There is a

no significant difference between the pre-op and post-op adjacent level disc disc angle. The mean adjacent level disc angle pre-op of 10.65 and the mean adjacent level disc height post-op of 8.76 (Table 8).

Table 8: Mean and Standard Deviation of Pre-op and Post-op Adjacent Level Disc Angle.

\begin{tabular}{|c|c|c|c|c|}
\hline Adjacent Disc Angle & N & Mean (0) & Std. Deviation \\
\hline Pre-op & 17 & 10.65 & 3.04 \\
\hline Post-op & 17 & 8.76 & 0.74 \\
\hline
\end{tabular}

\section{Complications}

No spinous process fracture occurred during implantation and there was no other intra-operative complications related to DIAM implant insertion noted. No patients was lost during follow-up.

\section{Discussion}

Device for intervertebral assisted motion or DIAM (Medtronic Sofamor Danec, Memphis, TN) is an example of a newly emerging concept of posterior dynamic stabilization interspinous device. The aim of this device is to restore or maintain segmental stability without arthrodesis [10,11]. The indications of placement of an interspinous device is poorly defined. DIAM were implanted for a variety of indications, and these can be broadly classified into three groups. The first indication is for discogenic disease or degenerative disc disease, either primary or recurrent, with or without discectomy. It is controversial but gaining acceptance as a distinct cause of low back pain. It refers to a continuum of non-radicular pain disorders of degenerative origin. Specifically excluded are symptoms related to disc impingement on neural elements, facet-mediated back pain, and spinal deformities secondary to lumbar degenerative disc disease (eg spondylolisthesis, degenerative scoliosis) [12]. Patients typically presents with axial low back pain without radicular symptoms. Pain often more severe with flexion (bending, sitting). The second indication is for posterior disease resulting in central stenosis, foramina stenosis, facet disease, or ligamentous instability. All this are the results of lumbar disc herniation or lumbar spinal stenosis. Lumbar disc herniation may present itself with symptoms of low back pain, radicular pain or cauda equina syndrome. It is due to prolapse of the disc which causes impingement of the exiting nerve roots. Lumbar spinal stenosis is defined by reduction in dimensions of central or lateral lumbar spinal canal by either bony structure or soft tissue structures [13]. Bony structures causing the stenosis are facet osteophytes, posterior vertebral body osteophytes or spondylolisthesis. Soft tissue structures causing the stenosis are herniated or bulging discs, hypertrophy or buckling of the ligamentous flavum, or synovial facet cysts. The third indication is to protect from junction disease by implanting a DIAM above a fresh or existing lumbar fusion [11].

Decompressive surgery such as discectomy is a common procedure for disc herniation without instability and it has shown to have favourable outcome [14]. However the outcome usually deteriorates over time due to recurrent stenosis, recurrent disc herniation or instability. Reoperation rates after discectomy are reported to range from 6 to $23 \%$ depending on the duration of follow-up and type of disease $[15,16]$. A study by Yoon-Joon et al concluded that patients who had DIAM implantation for lumbar spinal stenosis or disc herniation will undergo a reoperation rate of $8 \%$ at 4 years after surgery at the same level [17]. Biomechanically a study was done by Phillips et al found that DIAM device was effective in stabilising the unstable segment, reducing the increased segmental flexion-extension and lateral bending motions observed after discectomy [3].

The decision to perform discectomy with or without interspinous device in patients with a disc herniation is controversial $[2,3,18]$. However, the presence of low back pain and degenerative disc disease can support the placement of an interspinous device [19]. Although the insertion of an interspinous device has become a relatively common procedure for the treatment of lumbar stenosis and degenerative disc disease [20], its concomitant use with standard discectomy is not clear given the lack of comparative studies $[4,5,21]$. In this study, we have examined outcomes, first for functional disability and alleviation of pain due to potential stabilisation by DIAM implant, and second for confirmation that 
kyphosis or alteration of disc height at the index level and adjacent disc after DIAM implantation. In general, patients treated with discectomy with DIAM implantation for prolapsed intervertebral disc pathology demonstrated favourable outcomes at 6 months.

The most prevalent DIAM implanted level we found in this study was L5/S1 followed by L4/L5. Previous investigators have reported fewer implants at the L5/S1 level with majority being at L4/L5 [2,5,11]. Differences in the level of implant between this study and previous trials may reflect the varied clinical indications for surgery and refinements of the technique of insertion DIAM device over time.

In this study, there is a significant improvement in low back pain, leg pain and functional outcome based on Oswestry Disability Index at subsequent follow-up intervals up to 6 months, compared to preoperative baseline. VAS scores showed statistically significant improvements at 2 weeks, 3 months and 6 months postoperative. This is consistent with a few studies which investigates pain outcomes after DIAM implantation. Guizzardi et al. [22] inserted DIAM implant in young patients with disc herniations and low back pain. They found out that at 12 to 24 months postoperatively, $90 \%$ had no significant low back pain and were able to resume work. Another study by Iod et al who performed single level L4/ L5 DIAM implantation in patients with foramina stenosis, moderate degenerative instability or recurrent disc prolapse, found out that at 1 year post-op, $70 \%$ of patients achieved complete low back pain and radicular pain relief [23]. Taylor et al. [11] in a retrospective review of 95 of 104 (91\%) patients whom underwent DIAM insertion for mild single level stenosis as an adjunct to direct decompression, found that $88.5 \%$ of patients reported improvement in pain symptoms using the Dallas Self-Questionnaire and a $63.1 \%$ decrease in analgesic usage. Fabrizi et al. [5] whom retrospectively reviewed 1315 consecutive patients who underwent DIAM implantation both with and without direct decompression between 2000 and 2008, found significant improvements in all mean VAS scores at all follow-up intervals. It is unclear in our study to determine if the pain relief is from discectomy or in the presence of DIAM. The efficacy of DIAM device as an adjunct to decompressive surgery has been challenged. A prospective study on 62 patients between 2002 and 2006 was done by Kim et al. [21] In this study, 31 patients underwent laminectomy and/or microdiscectomy alone, while the other 31 patients underwent placement of DIAM implant in addition to laminectomy and/or discectomy. These patients were assessed clinically using VAS pain score and MacNab outcomes. Preoperative and postoperative MRI were used to measure disc height and sagittal alignment. They collectively found that there was no difference in VAS scores or MacNab outcomes in patients treated with or without DIAM implantation. They also found out that there is no difference in disc height between the treatment groups.

Radiologically, in our study, DIAM does not induce any significant change in index level disc height, intervertebral disc angle and adjacent segment disc height. The use of DIAM in patients with lordosis would theoretically increase posterior disc height and correct lordosis with kyphosis [24]. This is not seen in our study as the preoperative and postoperative mean disc height showed no statistical differences in disc height changes or change in lordosis (intervertebral disc angle) at index level. This results appear to be in agreement with the findings of Kim et al. [21] who used radiographic images taken with the patient in prone to assess their patients receiving decompression surgery augmented with a DIAM implant. They revealed an increase in mean lordosis of $1.20( \pm 0.24$; ratio range $0.97-1.450)$ at the index segment in the presence of DIAM; however this change was not statistically significant. Our study also revealed an increase in preoperative mean lordosis of $9.240( \pm 2.910)$ to $9.650( \pm 2.150)$ postoperative but was not statistically significant. The comparison between our study and Kim et al. [21] is questionable give the different methods of measurements used between these studies. Kim et al. [21] acknowledged that standing lateral radiographs would be more suitable imaging of sagittal alignment parameters for assessing the effect of DIAM surgery.

There is also no statistical differences in adjacent segment disc height changes $(11.33 \pm 2.53$ pre-op, $11.07 \pm 1.77$ post-op) and adjacent intervertebral disc angle $(10.65 \pm 3.04$ pre-op, $8.76 \pm 3.09$ post-op). Few biomechanical studies have reported that DIAM causes significant reduction in range of motion, especially in extension and flexion, whereas at the adjacent levels, no significant changes occurred [24,25]. These biomechanics advantages are expected to avoid development of adjacent level problems caused by fusion. Yoon-Joon et al. [17] who studied the survivorship of DIAM implant in 150 consecutive patients concluded that at 4 years after DIAM implantation, none of the patients underwent reoperation for adjacent level complications.

\section{Limitations}

There are a number of limitations to our study. First, the sample size for this study is small with a short follow-up of up to 6 months. Secondly, there is no control group which will give a better comparative results in the same cohort setting.

\section{Conclusion}

Device for intervertebral assisted motion or DIAM represents an alternative option to spinal fusion allowing motion preservation and restoration of mechanical properties in a degenerate functional spinal unit. It also provides stability and avoids potential degenerations at the adjacent levels. The placement of DIAM interspinous implant after discectomy showed improvements in VAS for leg pain and improvements in functional ability based on ODI at 6 months follow-up interval. A longer follow-up period is needed but early clinical results are good and promising.

\section{References}

1. JPG Urban, Roberts S (2003) Degeneration of intervertebral discs. Arthritic Research and Therapy 5(3): 120-130.

2. Benzel EC, Mroz T (2010) Interlaminar spacers: looks good, smells bad. World Neurosurg 74(6): 576-578. 
3. Phillips FM, Voronov LI, Gaitanis IN, Carandang G, Havey RM, et al. (2006) Biomechanics of posterior dynamic stabilizing device (DIAM) after facetectomy and discectomy. Spine J 6: 714-722.

4. Resnick DK (2007) Evidence-based spine surgery. Spine 32: S15-S19.

5. Fabrizi AP, Maina R, Schiabello L (2011) Interspinous spacers in the treatment of degenerative lumbar spinal disease: our experience with DIAM and Aperius devices. Eur Spine J 20: S20-S26.

6. Alfieri A, Gazzeri R, Prell J (2012) Role of lumbar interspinous distraction on the neural elements. Neurosurgical review 35(4): 477-484.

7. McCormack HM, Horne DJ, Sheather S (1988) Clinical applications of visual analogue scales: a critical review. Psychol Med 18(4): 1007-1119.

8. Fairbank JCT, Couper J, Davies JB, O’Brien JP (1980) The Oswestry low back pain disability questionnaire. Physiotherapy 66(8): 271-273.

9. Frobin W, Brinckmann P, Biggemann M, Tillotson M, Burton K (1997) Precision measurement of disc height, vertebral height and sagittal place displacement from lateral radiographic views of the lumbar spine. Clin Biotech 12(Suppl 1): S1-S63.

10. Luiz Henrique, Silvio RG (2006) Lumbar lordosis:a study of angle values and of vertebral bodies and intervertebral discs role. Acta Ortho Bra 14(4).

11. Taylor J, Pupin P, Delajoux S, Palmer S (2007) Device for Intervertebral Assisted Motion: Technique and Initial Results. Neurosurg Focus 22(1): E6.

12. Vincent J Devlin Discogenic Low Back Pain. Spine Secrets Plus, ( $2^{\text {nd }}$ edn), Elsevier, Netherlands, p. 337.

13. Derek Moore Lumbar Spinal Stenosis. Orthobullets.

14. Anjarwalla NK, Brown LC, McGregor AH (2007) The outcome of spinal decompression surgery 5 years on. Eur Spine J 16(11): 1842-1847.

15. Davis R (1994) A long-term outcome analysis of 984 surgically treated herniated lumbar discs. J Neurosurg 80(3): 415-421.

16. Wera GD, Marcus RE, Ghanayem AJ (2008) Failure within 1 year following subtotal lumbar discectomy. J Bone Joint Surg Am 90(1): 10-15.
17. Yoon Joon S, Chae Gwan K, Jong Beom P (2011) Survivorship analysis of 150 consecutive patients with DIAM implantation from surgery of lumbar spinal stenosis and disc herniation. Eur Spine J 20(2): 280-288.

18. Bono CM, Vaccaro AR (2007) Interspinous process devices in the lumbar spine. Contemporary Spine Surgery 8(9): 1-7.

19. Christie SD, Song JK, Fessler RG (2005) Dynamic interspinous process technology. Spine 30(16 Suppl): S73-S78.

20. Zucherman JF, Hsu KY, Hartjen CA, Mehalic TF, Implicito DA, et al. (2005) A multicenter, prospective, randomized trial evaluating the X STOP interspinous process decompression system for the treatment of neurogenic intermittent claudication: two-year follow-up results. Spine 30(12): 1351-1358.

21. Kim KA, McDonald M, Pik JHT (2007) Dynamic interspinous spacer technology for posterior stabilization: case control study on the safety, sagittal angulation, and pain outcome at 1-year follow-up evaluation. Neurosurg Focus 22(1): E7.

22. Guizzardi G, Alessandris A, Petrini P (2003) The use of DIAM in the prevention of chronic low back pain in young patients operated on for large dimension lumbar disc hernia. Presented at the $2^{\text {nd }}$ European Congress of Neurosurgery (EANS), Lison, Portugal 2003 (abstract) Rome, Meduzzi Editore, Italy.

23. Iob I, Fornasar G, Annusta P (2001) Axial-loaded CT SCN and DIAM implants in patients with failed back surgery syndrome. Presented at the Congress of Neurological Surgeons $51^{\text {st }}$ Annual Meeting, San Diego, California, USA

24. Barbagallo G, Alessandrello R, Barone F, Fricia M, Guiffrida M, et al (2002) DIAM: a new soft intervertebral implant for low-back pain treatment. Spine J 11(2 Suppl).

25. Sobottke R, Schluter-Brust K, Kaulhausen T (2009) Interspinous implants (X Stop, Wallis, DIAM) for the treatment of LSS: is there correlation between radiological parameters and clinical outcome? Euro Spine J 18(10): 1494-1503.
Creative Commons Attribution 4.0 International License

For possible submissions Click Here

\section{Submit Article}

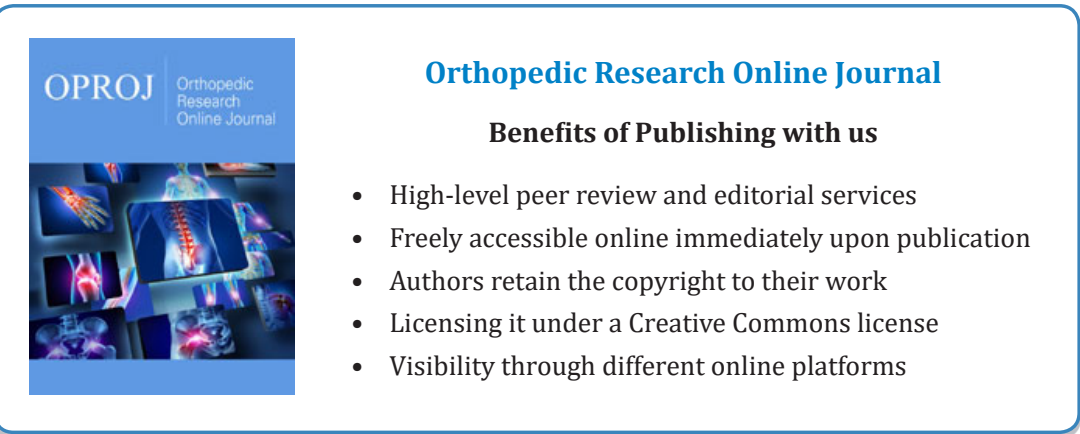

